PROCEEDINGS OF THE

AMERICAN MATHEMATICAL SOCIETY

Volume 126, Number 12, December 1998, Pages 3763-3765

S 0002-9939(98)04526-2

\title{
POLISHNESS OF THE WIJSMAN TOPOLOGY REVISITED
}

\author{
LÁSZLÓ ZSILINSZKY \\ (Communicated by Alan Dow)
}

\begin{abstract}
Let $X$ be a completely metrizable space. Then the space of nonempty closed subsets of $X$ endowed with the Wijsman topology is $\alpha$ favorable in the strong Choquet game. As a consequence, a short proof of the Beer-Costantini Theorem on Polishness of the Wijsman topology is given.
\end{abstract}

Denote by $C L(X)$ the nonempty closed subsets of the metric space $(X, d)$. The Wijsman topology $\tau_{W}$ on $C L(X)$ is the weak topology generated by $\{d(x, \cdot): x \in$ $X\}$, where the distance functional $d(x, A)=\inf \{d(x, a): a \in A\}$ is viewed as a function of the set argument $A \in C L(X)$. It was shown by G. Beer in [Be1] (see also $[\mathrm{Be} 2])$ that, given a separable complete metric space $(X, d)$, the corresponding hyperspace $\left(C L(X), \tau_{W}\right)$ is Polish. Since even uniformly equivalent metrics on $X$ may give rise to different Wijsman topologies (cf. [LL], [CLZ]), it required a separate argument to show that Polish base spaces always generate Polish Wijsman topologies. This was established by $\mathrm{C}$. Costantini in [Co1]. Thus, combining these results and the fact that $X$ embeds in $\left(C L(X), \tau_{W}\right)$ as a closed subspace, one gets

Theorem 1. The space $\left(C L(X), \tau_{W}\right)$ is Polish if and only if $(X, d)$ is Polish.

It is the purpose of this note to present a short proof of the above theorem based on the so-called strong Choquet game (cf. [Ch] or [Ke]). In the game, denoted by $\Gamma$, two players $\alpha$ and $\beta$ take turns in choosing objects in the topological space $X$ with an open base $\mathcal{B}: \beta$ starts by picking $\left(x_{0}, V_{0}\right)$ from $\mathcal{E}(X, \mathcal{B})=\{(x, V) \in X \times \mathcal{B}$ : $x \in V\}$ and $\alpha$ responds by picking $U_{0} \in \mathcal{B}$ with $x_{0} \in U_{0} \subset V_{0}$. The next choice of $\beta$ is some couple $\left(x_{1}, V_{1}\right) \in \mathcal{E}(X, \mathcal{B})$ with $V_{1} \subset U_{0}$, and again $\alpha$ picks $U_{1}$ with $x_{1} \in U_{1} \subset V_{1}$, etc. Player $\alpha$ wins the run $\left(x_{0}, V_{0}\right), U_{0}, \ldots,\left(x_{n}, V_{n}\right), U_{n}, \ldots$ provided $\bigcap_{n} U_{n}=\bigcap_{n} V_{n} \neq \emptyset$; otherwise $\beta$ wins. A winning tactic (abbr. w.t.) for $\alpha$ (cf. $[\mathrm{Ch}])$ is a function $\sigma: \mathcal{E}(X, \mathcal{B}) \rightarrow \mathcal{B}$ such that $\alpha$ wins every run of $\Gamma$ compatible with $\sigma$, i.e. such that $U_{n}=\sigma\left(x_{n}, V_{n}\right)$ for all $n$. The game $\Gamma$ is $\alpha$-favorable if $\alpha$ possesses a winning tactic; in this case $X$ is called a strong Choquet space (see $[\mathrm{Ke}]$ ). The Choquet Theorem (see [Ch], Theorem 8.7 or [Ke], Theorem 8.17) claims that a metrizable space is completely metrizable if and only if it is a strong Choquet space.

In the sequel $\omega$ will stand for the nonnegative integers, $B(x, \varepsilon)$ for the closed ball about $x \in X$ of radius $\varepsilon$ in the metric space $(X, d)$, and $B^{c}$ for the complement of

Received by the editors January 20, 1997 and, in revised form, April 28, 1997.

1991 Mathematics Subject Classification. Primary 54B20.

Key words and phrases. Wijsman topology, strong Choquet game, strong Choquet space, Polish space. 
$B \subset X$. For $U \subset X$ put $U^{-}=\{A \in C L(X): A \cap U \neq \emptyset\}$. It is routine to show that a base $\mathcal{B}_{W}$ for the Wijsman topology consists of the sets of the form

$$
\left(V_{0}, \ldots, V_{k}\right)_{D}=\bigcap_{i \leq k} V_{i}^{-} \cap \bigcap_{j \leq m}\left\{A \in C L(X): d\left(x_{j}, A\right)>\varepsilon_{j}\right\}
$$

with $V_{i} \subset X$ open $(i \leq k), x_{j} \in X, \varepsilon_{j}>0(j \leq m), D=\left(x_{0}, \ldots, x_{m} ; \varepsilon_{0}, \ldots, \varepsilon_{m}\right)$. For every $D$ of this kind, denote $M(D)=\bigcup_{j \leq m} B\left(x_{j}, \varepsilon_{j}\right)$.

Since metrizability of the Wijsman topology is equivalent to separability of the base space $X$ (see [Be2], Theorem 2.1.5), in order to prove Theorem 1 it suffices by the Choquet Theorem to prove

Theorem 2. If $X$ is completely metrizable, then $\left(C L(X), \tau_{W}\right)$ is a strong Choquet space.

Proof. Assume that $(X, \tau)$ is completely metrizable and $d$ is a compatible metric on $X$. Then by the Choquet Theorem we can find a w.t. $\sigma: \mathcal{E}(X, \tau) \rightarrow \tau$ for $\alpha$. Define a tactic $\sigma_{W}: \mathcal{E}\left(C L(X), \mathcal{B}_{W}\right) \rightarrow \mathcal{B}_{W}$ for $\alpha$ as follows: first, for each $V \in \tau$ and $A \in V^{-}$fix a point $x_{A, V} \in A \cap V$. Then, given $(A, \mathbf{V}) \in \mathcal{E}\left(C L(X), \mathcal{B}_{W}\right)$ with $\mathbf{V}=\left(V_{0}, \ldots, V_{k}\right)_{D}$ and $D=\left(x_{0}, \ldots, x_{m} ; \varepsilon_{0}, \ldots, \varepsilon_{m}\right)$, define

$$
\sigma_{W}(A, \mathbf{V})=\left(\sigma\left(x_{A, V_{0} \cap M(D)^{c}}, V_{0} \cap M(D)^{c}\right), \ldots, \sigma\left(x_{A, V_{k} \cap M(D)^{c}}, V_{k} \cap M(D)^{c}\right)\right)_{\tilde{D}}
$$

where $\tilde{D}=\left(x_{0}, \ldots, x_{m} ; \tilde{\varepsilon}_{0}, \ldots, \tilde{\varepsilon}_{m}\right)$ with $\tilde{\varepsilon}_{j}=\frac{\varepsilon_{j}+d\left(x_{j}, A\right)}{2}$ for all $j \leq m$. Then $A \in \sigma_{W}(A, \mathbf{U}) \subset \mathbf{U}$. We will show that $\sigma_{W}$ is a winning tactic for $\alpha$.

Indeed, suppose that $\left(A_{0}, \mathbf{V}_{0}\right), \mathbf{U}_{0}, \ldots,\left(A_{n}, \mathbf{V}_{n}\right), \mathbf{U}_{n}, \ldots$ is a run of $\Gamma$ in $C L(X)$ such that $\mathbf{U}_{n}=\sigma_{W}\left(A_{n}, \mathbf{V}_{n}\right)$ for all $n$. Denote $\mathbf{U}_{n}=\left(U_{0}^{n}, \ldots, U_{l_{n}}^{n}\right)_{B_{n}}$ and $\mathbf{V}_{n}=$ $\left(V_{0}^{n}, \ldots, V_{k_{n}}^{n}\right)_{D_{n}}$ for appropriate $B_{n}$ and $D_{n}$. Observe that $\mathbf{V}_{n+1} \subset \mathbf{U}_{n}$ and $\mathbf{V}_{n+1} \neq$ $\emptyset$ imply that $M\left(D_{n+1}\right)^{c} \subset M\left(B_{n}\right)^{c}$ and for all $s \leq l_{n}$ there exists $t \leq l_{n+1}$ such that $V_{t}^{n+1} \cap M\left(D_{n+1}\right)^{c} \subset U_{s}^{n} \cap M\left(B_{n}\right)^{c}$. Hence, without loss of generality, assume that $k_{n+1}>l_{n}=k_{n}$ and $t=s$. Put $l_{-1}=-1$. Then for all $n \in \omega$ and $l_{n-1}<i \leq l_{n}$,

$$
\begin{gathered}
\left(x_{A_{n}, V_{i}^{n} \cap M\left(D_{n}\right)^{c}}, V_{i}^{n} \cap M\left(D_{n}\right)^{c}\right), U_{i}^{n} \cap M\left(B_{n}\right)^{c}, \ldots, \\
\left(x_{A_{n+j}, V_{i}^{n+j} \cap M\left(D_{n+j}\right)^{c}}, V_{i}^{n+j} \cap M\left(D_{n+j}\right)^{c}\right), U_{i}^{n+j} \cap M\left(B_{n+j}\right)^{c}, \ldots
\end{gathered}
$$

is a run of $\Gamma$ in $X$ compatible with $\sigma$, so there exists $a_{i} \in \bigcap_{j \in \omega} U_{i}^{n+j} \cap M\left(B_{n+j}\right)^{c}$. Denote by $A$ the closure of $\left\{a_{i}: i \in \omega\right\}$ in $X$. Fix $n$ and $i$. Then for some $N>n$, $a_{i} \in U_{i}^{N} \cap M\left(B_{N}\right)^{c} \subset M\left(B_{n+1}\right)^{c}=M\left(\tilde{D}_{n}\right)^{c}$.

Consequently, if $D_{n}=\left(x_{0}, \ldots, x_{m} ; \varepsilon_{0}, \ldots, \varepsilon_{m}\right)$, then $d\left(x_{j}, a_{i}\right) \geq \tilde{\varepsilon}_{j}$ for all $j \leq m$; thus $d\left(x_{j}, A\right)=\inf \left\{d\left(x_{j}, a_{i}\right): i \in \omega\right\} \geq \tilde{\varepsilon}_{j}>\varepsilon_{j}$. It follows now that $A \in \mathbf{V}_{n}$ for all $n \in \omega$, whence $\alpha$ wins the run.

Remark. It is known that metrizable spaces that are not $\beta$-favorable in $\Gamma$ are the hereditarily Baire spaces (see [De]), i.e. spaces, every closed subspace of which is a Baire space. In general, however, even strong Choquet spaces may be nonhereditarily Baire $([\mathrm{De}])$. It could therefore be of interest to find out if complete metrizability of $X$ implies hereditary Baireness of $\left(C L(X), \tau_{W}\right)$. Note that it will certainly be a Baire space $([\mathrm{Zs}])$ and it could be non-Čech-complete, as was shown in $[\mathrm{Co} 2]$. 


\section{REFERENCES}

[Be1] G.Beer, A Polish topology for the closed subsets of a Polish space, Proc. Amer. Math. Soc. 113 (1991), 1123-1133. MR 92c:54009

[Be2] G.Beer, Topologies on Closed and Closed Convex Sets, Kluwer, Dordrecht, 1993. MR 95k:49001

[Co1] C.Costantini, Every Wijsman topology relative to a Polish space is Polish, Proc. Amer. Math. Soc. 123 (1995), 2569-2574. MR 95j:54012

[Co2] C.Costantini, On the hyperspace of a non-separable metric space, Proc. Amer. Math. Soc., to appear.

[Ch] G.Choquet, Lectures on Analysis I., Benjamin, New York, 1969. MR 40:3252

[CLZ] C.Costantini-S.Levi-J.Zieminska, Metrics that generate the same hyperspace convergence, Set-Valued Analysis 1 (1993), 141-157. MR 94h:54010

[De] G.Debs, Espaces héréditairement de Baire, Fund. Math. 129 (1988), 199-206. MR 90a:54082

[Ke] A.S.Kechris, Classical Descriptive Set Theory, Springer, New York, 1995. MR 96e:03057

[LL] A.Lechicki-S.Levi, Wijsman convergence in the hyperspace of a metric space, Boll. Un. Mat. Ital. (7) B.1 (1987), 439-451. MR 88e:54007

[Zs] L.Zsilinszky, Baire spaces and hyperspace topologies, Proc. Amer. Math. Soc. 124 (1996), 2575-2584. MR 96j:54017

Department of Mathematics, University of South Carolina, Columbia, South CarOLINA 29208

E-mail address: zsilinsz@math.sc.edu

Current address: Department of Mathematics and Computer Science, University of North Carolina at Pembroke, Pembroke, North Carolina 28372

E-mail address: laszlo@nat.uncp.edu 